Orthopäde $2019 \cdot 48: 175$

https://doi.org/10.1007/s00132-019-03689-7

Online publiziert: 21. Januar 2019

(c) Springer Medizin Verlag $\mathrm{GmbH}$, ein Teil von Springer Nature 2019

CrossMark

T. R. Blattert ${ }^{1}$ K. J. Schnake 2 0. Gonschorek ${ }^{3}$ S. Katscher ${ }^{4} \cdot$ B. W. Ullrich ${ }^{5}$.

E. Gercek ${ }^{6} \cdot$ F. Hartmann ${ }^{6}$ - S. Mörk' $\cdot$ R. Morrison ${ }^{8} \cdot$ M. L. Müller ${ }^{9}$ A. Partenheimer ${ }^{10}$. S. Piltz ${ }^{11} \cdot$ M. A. Scherer ${ }^{12} \cdot$ A. Verheyden ${ }^{13} \cdot$ V. Zimmermann $^{14} \cdot$ Sektion Wirbelsäule der Deutschen Gesellschaft für Orthopädie und Unfallchirurgie

'Orthopädische Fachklinik Schwarzach, Schwarzach, Deutschland

${ }^{2}$ Schön Klinik Nürnberg Fürth, Fürth, Deutschland

${ }^{3}$ Berufsgenossenschaftliche Unfallklinik Murnau, Murnau, Deutschland

${ }^{4}$ Interdisziplinäres Wirbelsäulenzentrum, Sana Klinikum Borna, Borna, Deutschland

${ }^{5}$ Klinik für Unfall- und Wiederherstellungschirurgie, BG Klinikum Bergmannstrost, Halle, Deutschland

${ }^{6}$ Zentrum für Unfallchirurgie und Orthopädie, Gemeinschaftsklinikum Mittelrhein, Koblenz, Deutschland

${ }^{7}$ St. Anna Krankenhaus, Sulzbach-Rosenberg, Deutschland

${ }^{8}$ Sektion konservative und operative Wirbelsäulentherapie, Klinikum Ingolstadt, Ingolstadt, Deutschland

${ }^{9}$ Klinik für Orthopädie und Unfallchirurgie, Universitätsklinikum Schleswig-Holstein, Kiel, Deutschland

${ }^{10}$ Spine \& Sport, Hannover, Deutschland

${ }^{11}$ Abteilung für Orthopädie und Unfallchirurgie, Klinikum Coburg gGmbH, Coburg, Deutschland

${ }^{12}$ Abteilung für Orthopädie und Unfallchirurgie, HELIOS Amper Klinikum Dachau, Dachau, Deutschland

${ }^{13}$ Klinik für Unfall-, Orthopädische und Wirbelsäulenchirurgie, Ortenauklinikum Lahr-Ettenheim, Lahr, Deutschland

${ }^{14}$ Zentrum für Hand- und Wirbelsäulenchirurgie, Klinikum Traunstein, Traunstein, Deutschland

\title{
Erratum zu: Nichtoperative und operative Behandlung der osteoporotischen Wirbelkörperfraktur
}

\section{Empfehlungen der Sektion Wirbelsäule der Deutschen Gesellschaft für Orthopädie und Unfallchirurgie (DGOU)}

Erratum zu:

Orthopäde 2018

https://doi.org/10.1007/s00132-018-

03666-6

Sehr geehrter Leser,

sehr geehrte Leserin,

bei der Online-First-Version des Beitrags Nichtoperative und operative Behandlung der osteoporotischen Wirbelkörperfraktur. Empfehlungen der Sektion Wirbelsäule der Deutschen Gesellschaft für Orthopädie und Unfallchirurgie (DGOU) wurde der Vorname des Co-Autors F. Hartmann leider falsch angegeben. Bitte beachten Sie die korrekte Nennung.
Wir bitten für den Fehler um Verzeihung. Der Originalbeitrag wurde korrigiert.

Die Redaktion

\section{Korrespondenzadresse}

Prof. Dr. T. R. Blattert

Orthopädische Fachklinik Schwarzach

Dekan-Graf-Straße 2-6, 94374 Schwarzach,

Deutschland

thomas.blattert@ofks.de

Die Online-Version des Originalartikels ist unter https://doi.org/10.1007/s00132-018-03666-6 zu finden. 